\title{
Bronchoalveolar lavage neuregulin-1 is elevated in acute lung injury and correlates
} with inflammation

\author{
James H. Finigan*, , Rangnath Mishra*, Vihas T. Vasu*, Lori J. Silveira*, \\ David E. Nethery", Theodore J. Standiford ${ }^{+}$, Ellen L. Burnham ${ }^{\#}$, \\ Marc Moss" ${ }^{\#}$ and Jeffrey A. Kern*,\#
}

ABSTRACT: Shedding of neuregulin (NRG)-1 from the pulmonary epithelium leads to activation of the epithelial human epidermal growth factor receptor (HER)2 receptor, increased pulmonary epithelial permeability and acute lung injury (ALI). We sought to determine whether NRG-1 was detectable and elevated in bronchoalveolar lavage (BAL) and plasma from patients with $A L I$ compared with controls and to determine whether a correlation exists between NRG-1 and inflammation and outcome in ALI.

Matched BAL and plasma samples were obtained from $23 \mathrm{ALI}$ patients requiring intubation and mechanical ventilation. Control patients $(n=5)$ included healthy volunteers. NRG-1 and indices of inflammation were measured in BAL and plasma via ELISA.

The mean \pm SD BAL NRG-1 concentration in ALI patients was $187.0 \pm 21.35 \mathrm{pg} \cdot \mathrm{mL}^{-1}$ compared with $85.50 \pm 9.2 \mathrm{pg} \cdot \mathrm{mL}^{-1}$ in controls $(\mathrm{p}=0.001)$. Increased BAL NRG-1 was associated with markers of inflammation, and inversely correlated with ventilator-free days (VFDs; $r=-0.51, p=0.015$ ). Plasma NRG-1 was elevated in ALI patients compared with controls $(611.7 \pm 354.2$ versus $\left.25.17 \pm 19.33 \mathrm{pg} \cdot \mathrm{mL}^{-1}, \mathrm{p}<0.001\right)$ and inversely correlated with VFDs $(r=-0.51, p=0.04)$

These results confirm shedding of NRG-1 in ALI and suggest that the NRG-1-HER2 pathway is active in patients with ALI.

KEYWORDS: Acute respiratory failure, alveolar epithelium, biomarkers, inflammatory mediators

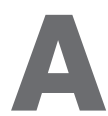
cute lung injury (ALI) and its more severe manifestation, the acute respiratory distress syndrome (ARDS) affects up to 200,000 people in the USA each year and has a mortality rate of up to $40 \%$ [1]. Despite recent advances in the understanding of the pathogenic mechanisms of ALI, no specific therapy exists and treatment remains supportive. Epithelial injury is the pathological hallmark of ALI [2, 3], and damage to the pulmonary epithelium by inflammation and inflammatory cytokines is an initiating step in ALI development [4]. The overall effect is increased paracellular permeability of the alveolar epithelium resulting in pulmonary oedema and life-threatening hypoxaemia [4]. The pulmonary alveolar epithelium, composed of type I and type II cells, is a critical participant in the pathogenesis of pulmonary oedema development in ALI, and an intact epithelial barrier is essential to maintaining normal pulmonary fluid balance [5-7].
The human epidermal growth factor receptor (HER)2, a receptor tyrosine kinase (RTK) expressed by bronchial and alveolar epithelial cells, is involved in multiple physiological processes including cell proliferation, cell-cell adhesion and epithelial permeability. The HER receptor family consists of four type 1, membrane-bound, RTKs: HER1 or epidermal growth factor receptor (EGFR), and HER2, -3 and -4 [8]. Receptor signalling occurs via ligand binding, which induces receptor homoor heterodimerisation, activation of the receptor kinase domain, autophosphorylation and initiation of downstream signalling. HER2 has no known ligand and requires partnering with another HER family member for activation. While HER3 has no intrinsic signalling properties, it is the receptor for the growth factor ligand neuregulin (NRG)-1 [9], and its preferred co-receptor is HER2. NRG-1 is cells [10-13], and is shed from the epithelial cell expressed in bronchial and alveolar epithelial
AFFILIATIONS

*Dept of Medicine, National Jewish Health,

\#Division of Pulmonary Sciences and Critical Care Medicine, University of Colorado, Denver, CO,

"Division of Pulmonary, Critical Care and Sleep Medicine, University Hospitals Case Medical Center, Case Western Reserve University,

Cleveland, $\mathrm{OH}$, and

+University of Michigan, Ann Arbor, MI, USA.

CORRESPONDENCE

J.H. Finigan

Dept of Medicine

National Jewish Health

1400 Jackson Street

K736A

Denver

CO 80206

USA

E-mail: Finigan」@NJHealth.org

Received:

Jan 092012

Accepted after revision:

May 042012

First published online:

May 172012 
surface by the protease a disintegrin and metalloprotease (ADAM)17 to bind HER3 in an auto/paracrine fashion [10, 14]. This results in HER2/HER3 receptor complex formation and HER2 activation [11]

Our previous findings demonstrated that the inflammatory cytokine interleukin (IL)-1 $\beta$ induces ADAM17-mediated NRG-1 shedding and subsequent HER2 activation leading to increased pulmonary epithelial cell permeability in vitro [10]. Animal models of ALI identified activation of this pathway in vivo [10, $15,16]$. Consistent with enhanced epithelial NRG-1 shedding in the setting of lung injury, NRG-1 was detected in pulmonary oedema fluid from a small group of ALI patients and was elevated compared with patients with cardiogenic pulmonary oedema [10], further suggesting a role for this pathway in disease. However, it is unknown whether NRG-1 levels correlate with pulmonary inflammation and outcome, and whether NRG-1 shedding is compartmentalised to the lung.

Given the role of the NRG-1-HER2 pathway in ALI models and evidence of increased NRG-1 shedding in experimental models of inflammatory lung injury, we hypothesised that NRG-1 levels in bronchoalveolar lavage (BAL) samples from patients with ALI would be elevated compared with healthy controls. We also sought to determine whether BAL NRG-1 levels in ALI patients would correlate with ALI severity and would be associated with increased pulmonary inflammation. Finally, we investigated whether NRG-1 would be elevated in plasma from ALI patients compared with healthy controls and would correlate with BAL NRG-1.

\section{MATERIALS AND METHODS Patients}

This study was reviewed by the National Jewish Health Institutional Review Board (Denver, CO, USA) and was determined not to involve "research on human subjects" or "protected health information" as defined by the US Dept of Health and Human Services (Bethesda, MD, USA). All biological samples and clinical data were pre-existing and obtained in a deidentified fashion from a subset of patients enrolled in the clinical study "A randomized trial of GM-CSF in patients with ALI/ARDS" (ClinicalTrials.gov identifier NCT00201409), a multicentre, randomised, placebo-controlled phase II study examining the efficacy of granulocyte-macrophage colonystimulating factor (GM-CSF) versus placebo for the treatment of ALI [17]. Patients included in our analysis were enrolled between July 2004 and June 2009, were $\geqslant 18$ yrs of age, met ALI criteria as defined by the 1994 American-European consensus statement [18], required endotracheal intubation and mechanical ventilation (MV), and underwent BAL and had blood collected on the third day after meeting ALI criteria. Patients who died prior to day 3 did not have samples collected and, therefore, were not included in our analysis. As one goal was to determine a correlation between BAL and plasma NRG-1 in early ALI, only patients with both BAL and plasma available on the third day after meeting ALI criteria were chosen for measurement of NRG-1 levels $(n=23)$. Healthy volunteers without lung disease who underwent BAL via bronchoscopy and donated plasma served as control subjects. BAL samples were filtered through sterile gauze to remove noncellular particulate material and mucus. Samples were then centrifuged $(1,000 \times g$ for $5 \mathrm{~min})$ after collection to separate the cells and supernatant. The supernatant was aliquoted into small volumes $(<10 \mathrm{~mL})$. Blood was similarly centrifuged to separate cells from plasma immediately after collection and aliquoted. All plasma and BAL supernatant samples were stored at $-80^{\circ} \mathrm{C}$.

\section{Clinical data}

Clinical information on all patients was collected as part of the GM-CSF study. Acute Physiology and Chronic Health Evaluation II (APACHE II) scores, Sequential Organ Failure Assessment (SOFA) scores and arterial oxygen tension $\left(\mathrm{Pa}_{1} \mathrm{O}_{2}\right) /$ inspiratory oxygen fraction $\left(\mathrm{F}, \mathrm{O}_{2}\right)$ ratios were calculated at enrolment (day 1 ALI). The primary outcome, ventilator-free days (VFDs), was defined as the number of days a patient was alive and free of MV out of 28. Intensive care unit (ICU)-free days and mortality (by 28 days) were also recorded.

\section{Biomarker measurement}

NRG-1 in BAL and plasma was measured by ELISA (R\&D Systems, Inc., Minneapolis, MN, USA). A panel of analytes including epidermal growth factor (EGF), IL-1 $\beta$, IL-6, IL-8 and tumour necrosis factor (TNF)- $\alpha$ were measured simultaneously in BAL, and plasma samples using a human cytokine 5-plex 96well assay kit (Millipore, Billerica, MA, USA) was run according to the manufacturer's instructions and measured using Luminex technology (Bio-plex, Bio-Rad, Hercules, CA, USA). All biological analytes were measured in duplicate. Investigators processing biological samples were blinded to the clinical data.

\section{Statistical analysis}

Summary statistics of the demographic information for the population were presented as either mean \pm SD in the continuous case or count (percentage of total) in the categorical case. The primary outcome variable in this study was VFDs. Secondary outcome variables were ICU-free days as well as enrolment APACHE II score, SOFA score and $\mathrm{Pa}_{2} \mathrm{O}_{2} / \mathrm{FI}_{1} \mathrm{O}_{2}$ ratio. Relationships between outcome variables and biological predictors were first investigated using Pearson product-moment correlations. All biological predictors were natural log transformed to comply with model assumptions. Bootstrapping of estimates was also performed in order to further strengthen our findings. Bootstrapping is a nonparametric technique that is used to develop the sampling distribution of the test statistic in order to evaluate an empirical standard error. In all cases, the bootstrapped empirical analysis coincided with the traditional

\begin{tabular}{lc}
\hline TABLE 1 & Baseline patient characteristics \\
\hline Males & $14(60.9)$ \\
Age $\mathbf{y r s}$ & $47.9 \pm 14.1$ \\
$\mathbf{P a}_{\mathbf{O}} / \mathbf{F I} \mathbf{O}_{2}$ ratio & $133.2 \pm 47.6$ \\
APACHE II score & $19.2 \pm 8.3$ \\
SOFA score & $6.3 \pm 3.8$ \\
Ventilator-free days & $12.4 \pm 8.3$ \\
ICU-free days & $8.0 \pm 8.1$ \\
Survival & $20(87)$
\end{tabular}

Data are presented as $\mathrm{n}(\%)$ or mean $\pm \mathrm{SD}$. $\mathrm{Pa}_{\mathrm{a}_{2}}$ : arterial oxygen tension; $\mathrm{Fl}_{1} \mathrm{O}_{2}$ : inspiratory oxygen fraction; APACHE II: Acute Physiology and Chronic Health Evaluation II; SOFA: Sequential Organ Failure Assessment; ICU: intensive care unit. ${ }^{\#}: n=23$. 


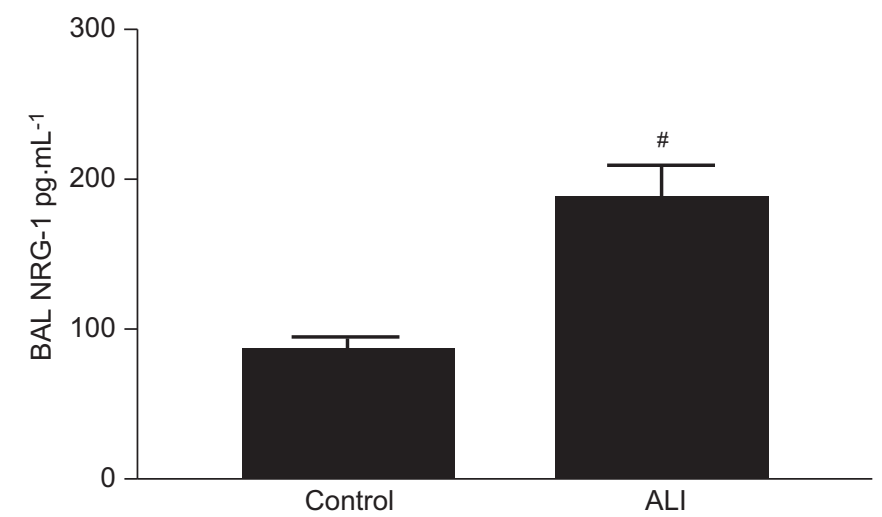

FIGURE 1. Bronchoalveolar lavage (BAL) levels of neuregulin (NRG)-1 in acute lung injury (ALI) compared with healthy controls. NRG-1 was measured in BAL from ALI patients $(n=23)$ via ELISA and compared with healthy volunteers $(n=5)$. The mean BAL NRG- 1 in ALI was $187.0 \pm 21.35 \mathrm{pg} \cdot \mathrm{mL}^{-1}$ compared with $85.50 \pm 9.2 \mathrm{pg} \cdot \mathrm{mL}^{-1}$ in controls. ${ }^{*}: p=0.001$.

parametric analysis. All analyses were performed using SAS 9.2 (SAS Institute Inc., Cary, NC, USA) or the R version 2.13.2 (The R Foundation for Statistical Computing, Vienna, Austria). All tests were two-sided and p-values of $<0.05$ were considered significant.

\section{RESULTS}

\section{BAL NRG-1 is elevated in ALI}

Patient characteristics at study entry are shown in table 1. On study entry (enrolment day 1), all patients met ALI $\mathrm{Pa}_{\mathrm{a}} \mathrm{O}_{2} / \mathrm{FI}_{1} \mathrm{O}_{2}$ criteria [18] with a mean $\pm \mathrm{SD} P \mathrm{~Pa}_{2} \mathrm{O}_{2} / \mathrm{FI}_{1} \mathrm{O}_{2}$ ratio of $133 \pm 47$. The average age was 47.9 yrs and $39 \%$ of the patients were female. The mean \pm SD enrolment APACHE II score was 19.2 \pm 8.3 and SOFA score was $6.3 \pm 3.8$. The average VFDs was $12.4 \pm 8.3$ and ICU-free days were $7.9 \pm 8.1$. All but three patients were alive at 28 days. 12 patients received GM-CSF and 11 received placebo. There was no difference in NRG-1 levels between patients receiving GM-CSF and placebo.

NRG-1 was detectable in the BAL of all ALI patients. The mean BAL NRG-1 concentration in patients with ALI was significantly higher than what was measured in controls $(187.0 \pm 21.35$ versus $85.50 \pm 9.20 \mathrm{pg} \cdot \mathrm{mL}^{-1}, \mathrm{p}=0.001$; fig. 1$)$. As IL- $1 \beta$ induces NRG-1 shedding in vitro [10], we sought to determine whether that NRG-1 shedding in ALI patients would occur concomitantly with alveolar inflammation. We therefore measured BAL indices of inflammation including IL- $1 \beta$, IL- 6 , IL- 8 and TNF- $\alpha$ to determine their correlation with NRG-1 (table 2). NRG-1 was positively and significantly correlated with IL-6 $(\mathrm{p}<0.05)$ and TNF- $\alpha(\mathrm{p}<0.05)$ in BAL. Increases in BAL NRG-1 were associated with increases in BAL IL-1 $\beta$ and IL-8, although this did not reach statistical significance $(p<0.06$ and $p<0.08$ for IL- $1 \beta$ and IL- 8 , respectively). These data demonstrate that NRG-1 is elevated in ALI compared with control subjects and that the NRG-1 concentration is associated with markers of inflammation.

\section{BAL NRG-1 does not correlate with EGF}

As others have reported signalling via EGF and its receptor, the EGFR, in ALI [19, 20], we sought to determine whether EGF was present in BAL from patients with ALI. While we did detect a low level of EGF in BAL from some patients with ALI (median $1.62 \mathrm{pg} \cdot \mathrm{mL}^{-1}$ ), there was no correlation between EGF and NRG-1 or VFDs (table 2).

\section{BAL NRG-1 correlates with ALI severity}

Given the overall low mortality in our cohort, we chose VFDs, a commonly used outcome variable that may reflect ALI severity, to determine any correlation between NRG-1 and clinical outcome in our analysis. As can be seen in table 3, higher BAL NRG-1 was associated with fewer VFDs $(r=-0.51$, $\mathrm{p}=0.015)$. In addition, increasing BAL NRG-1 was also negatively associated with the secondary outcome of ICU-free days $(\mathrm{r}=-0.50, \mathrm{p}=0.015)$. There was no correlation between BAL NRG-1 and enrolment $\mathrm{Pa}, \mathrm{O}_{2} / F \mathrm{I}_{1} \mathrm{O}_{2}$ ratio, APACHE II score or SOFA score (table 3 ).

\section{NRG-1 is elevated in plasma during ALI}

We evaluated BAL NRG-1 as a reflection of shedding from pulmonary epithelial cells. However, ALI is marked by a systemic inflammatory response that may lead to increased systemic NRG-1 concentration. We therefore assayed plasma from subjects with ALI for NRG-1 and compared these values to NRG-1 levels in BAL. As seen in figure 2, plasma NRG-1 was detected in all patients with ALI and significantly elevated compared with control subjects $\left(611.7 \pm 354.2 \mathrm{pg} \cdot \mathrm{mL}^{-1}\right.$ versus $\left.25.17 \pm 19.33 \mathrm{pg} \cdot \mathrm{mL}^{-1}, \mathrm{p}<0.001\right)$. In addition, plasma NRG-1 concentration significantly correlated with BAL NRG-1 with correlation coefficient $0.53(\mathrm{p}=0.05)$. Consistent with BAL NRG-1, higher plasma NRG-1 concentration was associated with longer duration of MV, with a correlation between plasma NRG-1 and VFD of -0.51 ( $\mathrm{p}=0.04)$. In contrast to BAL NRG-1, plasma NRG-1 concentration did not correlate with plasma

\begin{tabular}{|c|c|c|c|c|c|c|}
\hline & NRG-1 & EGF & IL-1 $\beta$ & IL-6 & IL-8 & TNF- $\alpha$ \\
\hline EGF & $-0.02(p=0.91)$ & & $-0.24(p=0.27)$ & $-0.17(p=0.42)$ & $0.25(p=0.26)$ & $-0.24(p=0.27)$ \\
\hline IL-1 $\beta$ & $0.40(p=0.06)$ & $-0.24(p=0.27)$ & & $0.58(p=0.0034)$ & $0.61(p=0.0019)$ & $0.86(p<0.0001)$ \\
\hline IL-6 & $0.41(p=0.05)$ & $-0.17(p=0.42)$ & $0.58(p=0.0034)$ & & $0.66(p=0.0007)$ & $0.81(p<0.0001)$ \\
\hline TNF- $\alpha$ & $0.45(p=0.03)$ & $-0.24(p=0.27)$ & $0.86(p<0.0001)$ & $0.81(p<0.0001)$ & $0.72(p<0.0001)$ & \\
\hline
\end{tabular}

NRG: neuregulin; EGF: epidermal growth factor; IL: interleukin; TNF: tumour necrosis factor. 
TABLE 3 Correlation coefficients between bronchoalveolar lavage growth factor and cytokine concentrations and outcomes

\begin{tabular}{lccccc} 
& VFDs & ICU-free days & APACHE II & SOFA & Pa, $\mathbf{O}_{2} / \mathbf{F}, \mathbf{O}_{\mathbf{2}}$ Score \\
\hline NRG-1 & $-0.51(p=0.015)$ & $-0.50(p=0.015)$ & $0.19(p=0.37)$ & $0.14(p=0.91)$ & $-0.02(p=0.91)$ \\
EGF & $0.31(p=0.17)$ & $0.44(p=0.038)$ & $-0.10(p=0.66)$ & $0.16(p=0.48)$ & $-0.13(p=0.55)$ \\
IL-1 $\beta$ & $-0.29(p=0.20)$ & $-0.17(p=0.43)$ & $0.12(p=0.59)$ & $0.01(p=0.95)$ & $0.21(p=0.34)$ \\
IL-6 & $-0.34(p=0.13)$ & $-0.22(p=0.32)$ & $0.06(p=0.79)$ & $0.26(p=0.23)$ & $0.005(p=0.98)$ \\
IL-8 & $-0.12(p=0.61)$ & $0.09(p=0.69)$ & $0.28(p=0.19)$ & $0.44(p=0.04)$ & $0.11(p=0.62)$ \\
TNF- $\boldsymbol{\alpha}$ & $-0.26(p=0.26)$ & $-0.15(p=0.49)$ & $0.12(p=0.60)$ & $0.09(p=0.68)$ & $0.19(p=0.40)$ \\
\hline
\end{tabular}

VFDs: ventilator-free days; ICU: intensive care unit; APACHE II: Acute Physiology and Chronic Health Evaluation II; SOFA: Sequential Organ Failure Assessment; $P_{a}, \mathrm{O}_{2}$ arterial oxygen tension; $\mathrm{Fl}_{1} \mathrm{O}_{2}$ : inspiratory oxygen fraction; NRG: neuregulin; EGF: epidermal growth factor; IL: interleukin; TNF: tumour necrosis factor.

levels of other inflammatory mediators or secondary outcomes (data not shown). EGF was detected in the plasma from nine out of 23 ALI patients but did not correlate with NRG-1 or with any outcome variables (data not shown).

\section{DISCUSSION}

This study demonstrates that NRG-1 is detectable in BAL and plasma from patients with ALI and is elevated compared with controls. In addition, BAL NRG-1 correlates with markers of pulmonary inflammation and outcomes including VFDs and ICU-free days. We have previously described the role of the NRG-1-HER2 signalling axis in epithelial dysfunction in vitro and lung injury in vivo $[10,15,16]$. Our current findings provide supportive evidence that NRG-1 shedding occurs in patients with ALI [10] and suggest that NRG-1 (BAL and/or plasma) might serve as a novel biomarker to provide prognostic information in ALI. A biomarker that predicts outcome, reflects disease pathophysiology and has existing treatment would be a major step forward in management of ALI.

That NRG-1 might serve as a biomarker in ALI is logical given the potential role of the NRG-1-HER2 pathway in ALI pathophysiology. Stimulation of pulmonary epithelial cells with IL-1 $\beta$ resulted in shedding of NRG-1, HER2 activation and

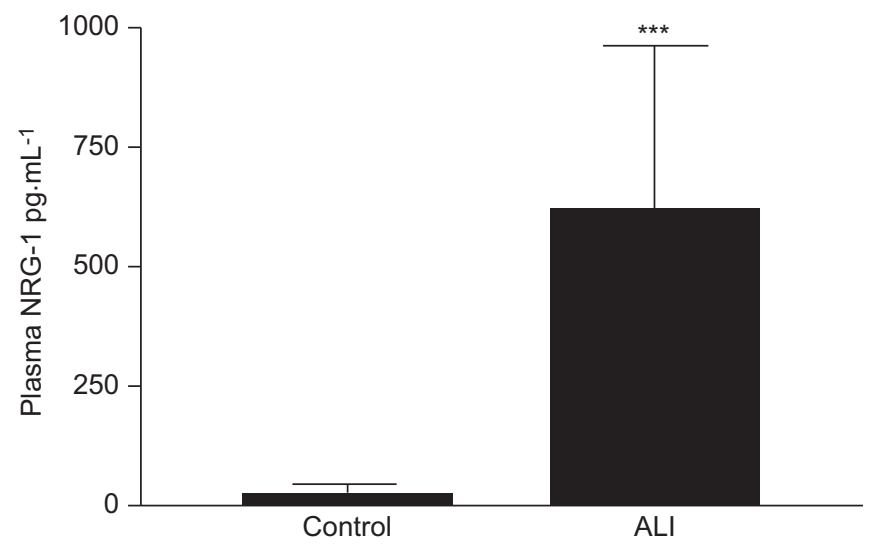

FIGURE 2. Plasma levels of neuregulin (NRG)-1 in acute lung injury (ALI) compared with healthy controls. NRG-1 was measured in plasma from ALI patients $(\mathrm{n}=23)$ via ELISA and compared with healthy volunteers $(n=5)$. The mean plasma NRG-1 in ALI was $611.7 \pm 354.2 \mathrm{pg} \cdot \mathrm{mL}^{-1}$ compared with $25.17 \pm 19.33 \mathrm{pg} \cdot \mathrm{mL}^{-1}$ in controls. ***: $p<0.001$
HER2-dependent increases in paracellular pulmonary epithelial permeability [10]. In mouse models of ALI, increased pulmonary leakage is associated with elevated BAL NRG-1 and increased pulmonary HER2 activation. Pharmacological and genetic strategies to downregulate HER2 activation attenuated ALI, decreased pulmonary fibrosis and improved survival in bleomycin lung injury models $[15,16]$. Similarly, inhibition of ADAM17, the protease that cleaves NRG-1 from the epithelial surface, attenuated pulmonary NRG-1 shedding, HER2 activation and ALI in mice [10]. A contribution to ALI pathophysiology with evidence of pathway activation in patients identifies ADAM17, NRG-1 and HER2 as possible therapeutic targets in ALI. Drug treatments of ALI are extremely limited. Confirmation that the ADAM17-NRG-1-HER2 pathway participates in ALI pathophysiology immediately opens the door to a host of existing pharmacological therapies targeting ADAM17, NRG-1 and HER2.

Our data identified a significant correlation between both BAL and plasma NRG-1 and VFDs. VFDs are a composite end-point that includes the time a patient is alive and free of MV in a 28day period and has become a commonly used and accepted pulmonary outcome in patients with ALI [21-23]. The relatively small number of patients studied and their low mortality rate $(13 \%)$ did not allow us to identify a correlation between 28-day survival and BAL or plasma NRG-1 concentration. However, an association with VFDs provides useful clinical information. Prolonged ventilator duration has been consistently demonstrated to be a risk factor for long-term complications of ALI, including impairment in activities of daily living [24], decreased 6-min walk distance [25], post-ALI anxiety and compromised long-term pulmonary function [26].

We identified increased NRG-1 in BAL and plasma, and that NRG-1 levels were higher in plasma than in BAL. The tissue source of plasma NRG-1 is unknown. Although there is high expression in the lung, other organs, such as the heart and liver, express NRG-1 [12], and it is possible that generalised inflammation induces shedding of NRG-1 from other tissue beds. It is not clear why plasma NRG-1 does not correlated with plasma cytokines and this requires further study. Given that inflammatory cytokines induce NRG-1 shedding, that BAL NRG-1 does correlate with pulmonary inflammation suggests that the BAL NRG-1 is from the lung, although translocation from the bloodstream cannot be excluded. It is notable that plasma NRG-1 correlated with BAL NRG-1 and VFDs, 
supporting ALI as a disease marked by generalised inflammation and suggesting that plasma NRG-1 might accurately reflect NRG-1 signalling in the lung and might serve as a novel biomarker for ALI. Serum biomarkers, particularly when used in combination and in conjunction with clinical parameters, are able to predict mortality $[27,28]$. In addition to providing prognostic information, a biomarker might be used to select patients for clinical trials of new therapies [29].

There are limitations of our study. Though NRG-1 was elevated in ALI patients compared with healthy volunteers, a more appropriate control group might include intubated, critically ill patients without lung injury, such as heart failure patients. However, usually identifying ALI is not a diagnostic challenge requiring biochemical confirmation. It is possible that NRG-1 might be elevated in other critically ill populations. There is often elevation of inflammatory cytokines in critically ill patients without lung injury, and cytokine levels can overlap with those obtained from ALI patients [30]. In addition, even non-injurious MV activates a pro-inflammatory transcriptional profile in the lung [31]. However, our data suggest that, in a patient with known ALI, NRG-1 levels have prognostic value.

While our sample size was similar to other studies investigating biomarker levels in BAL [32-34], it was underpowered to detect an association between NRG-1 and mortality. In addition, our low mortality rate might explain why we did not detect an association between NRG-1 and APACHE II or SOFA scores, given that these are used to predict mortality in critically ill patients. We did not identify an association between NRG-1 and oxygenation; however, oxygenation, particularly at onset of ALI, is a poor predictor of outcome [29]. However, our sample size was adequate to determine significance between both BAL and plasma NRG-1 and VFDs. VFDs are a composite outcome of time off a ventilator and mortality, and therefore observed differences in VFDs could reflect differences in mortality and not differences in ventilation duration [35]. The low death rate in our cohort suggests that the correlation between NRG-1 and VFDs is real and not influenced by mortality [29]. Samples collected over multiple time-points from a large and diverse population of lung injury patients (e.g. sepsis, trauma, etc.) is needed to definitively determine the utility of NRG-1 as a diagnostic and prognostic biomarker for ALI. Finally, though we observed NRG-1 shedding, HER2 activation was not directly assessed in humans. Given the low dissociation constant ( $\sim 100 \mathrm{pM})$ of NRG-1 for the HER2/3 complex, the presence of free NRG-1 would suggest that HER2 activation is likely. However, the possibility of a NRG-1 or HER2 inhibitor or an inactive form of NRG-1 is conceivable (though speculative) and would require study; our current findings support NRG-1 shedding as pathophysiological process in ALI.

ALI is a common and lethal inflammatory syndrome with no pharmacological treatments. Our ability to stratify patients by severity and predict outcome is limited, further compromising care of these patients. Our data indicate that the NRG-1-HER2 pathway is activated in patients with ALI and suggest that the NRG-1 concentration might be used as marker of inflammation and disease severity. Finally, elevated NRG-1 levels might serve as an indication for use of therapies targeting the sheddase (ADAM17), the ligand (NRG-1) or the receptor (HER2) in patients with ALI.

\section{SUPPORT STATEMENT}

This work was funded by NIH HL075422 and the VA Merit Award (both awarded to J.A. Kern), and NIH K08 HL086668 (awarded to J.H. Finigan).

\section{STATEMENT OF INTEREST}

None declared.

\section{REFERENCES}

1 Rubenfeld GD, Caldwell E, Peabody E, et al. Incidence and outcomes of acute lung injury. N Engl J Med 2005; 353: 1685-1693.

2 Bachofen M, Weibel ER. Alterations of the gas exchange apparatus in adult respiratory insufficiency associated with septicemia. Am Rev Respir Dis 1977; 116: 589-615.

3 Bachofen M, Weibel ER. Structural alterations of lung parenchyma in the adult respiratory distress syndrome. Clin Chest Med 1982; 3: $35-56$.

4 Ware LB, Matthay MA. The acute respiratory distress syndrome. N Engl J Med 2000; 342: 1334-1349.

5 Matthay MA. Function of the alveolar epithelial barrier under pathologic conditions. Chest 1994; 105: 67S-74S.

6 Inoue S, Michel RP, Hogg JC. Zonulae occludentes in alveolar epithelium and capillary endothelium of dog lungs studies with the freeze-fracture technique. J Ultrastruct Res 1976; 56: 215-225.

7 Gorin AB, Stewart PA. Differential permeability of endothelial and epithelial barriers to albumin flux. J Appl Physiol 1979; 47: 1315-1324.

8 Yarden Y, Sliwkowski MX. Untangling the ErbB signalling network. Nat Rev Mol Cell Biol 2001; 2: 127-137.

9 Kern JA, Robinson RA, Gazdar A, et al. Mechanisms of p185HER2 expression in human non-small cell lung cancer cell lines. Am J Respir Cell Mol Biol 1992; 6: 359-363.

10 Finigan JH, Faress JA, Wilkinson E, et al. Neuregulin-1-human epidermal receptor-2 signaling is a central regulator of pulmonary epithelial permeability and acute lung injury. J Biol Chem 2011; 286: 10660-10670.

11 Patel NV, Acarregui MJ, Snyder JM, et al. Neuregulin-1 and human epidermal growth factor receptors 2 and 3 play a role in human lung development in vitro. Am J Respir Cell Mol Biol 2000; 22: 432-440.

12 Holmes WE, Sliwkowski MX, Akita RW, et al. Identification of heregulin, a specific activator of p185erbB2. Science 1992; 256: 1205-1210.

13 Vermeer PD, Einwalter LA, Moninger TO, et al. Segregation of receptor and ligand regulates activation of epithelial growth factor receptor. Nature 2003; 422: 322-326.

14 Gollamudi M, Nethery D, Liu J, et al. Autocrine activation of ErbB2/ErbB3 receptor complex by NRG-1 in non-small cell lung cancer cell lines. Lung Cancer 2004; 43: 135-143.

15 Nethery DE, Moore BB, Minowada G, et al. Expression of mutant human epidermal receptor 3 attenuates lung fibrosis and improves survival in mice. J Appl Physiol 2005; 99: 298-307.

16 Faress JA, Nethery DE, Kern EF, et al. Bleomycin-induced pulmonary fibrosis is attenuated by a monoclonal antibody targeting HER2. J Appl Physiol 2007; 103: 2077-2083.

17 Paine R 3rd, Standiford TJ, Dechert RE, et al. A randomized trial of recombinant human granulocyte-macrophage colony-stimulating factor for patients with acute lung injury. Crit Care Med 2012; 40: 90-97.

18 Bernard GR, Artigas A, Brigham KL, et al. The AmericanEuropean Consensus Conference on ARDS. Definition, mechanisms, relevant outcomes, and clinical trial coordination. Am J Respir Crit Care Med 1994; 149: 818-824. 
19 Bierman A, Yerrapureddy A, Reddy NM, et al. Epidermal growth factor receptor (EGFR) regulates mechanical ventilation-induced lung injury in mice. Transl Res 2008; 152: 265-272.

20 Harada C, Kawaguchi T, Ogata-Suetsugu S, et al. EGFR tyrosine kinase inhibition worsens acute lung injury in mice with repairing airway epithelium. Am J Respir Crit Care Med 2011; 183: 743-510.

21 Schoenfeld DA, Bernard GR. Statistical evaluation of ventilatorfree days as an efficacy measure in clinical trials of treatments for acute respiratory distress syndrome. Crit Care Med 2002; 30: 1772-1777.

22 The Acute Respiratory Distress Syndrome Network. Ventilation with lower tidal volumes as compared with traditional tidal volumes for acute lung injury and the acute respiratory distress syndrome. The Acute Respiratory Distress Syndrome Network. N Engl J Med 2000; 342: 1301-1308.

23 Wiedemann HP, Wheeler AP, Bernard GR, et al. Comparison of two fluid-management strategies in acute lung injury. $N$ Engl J Med 2006; 354: 2564-2575.

24 van der Schaaf M, Beelen A, Dongelmans DA, et al. Poor functional recovery after a critical illness: a longitudinal study. J Rehabil Med 2009; 41: 1041-1048.

25 Herridge MS, Cheung AM, Tansey CM, et al. One-year outcomes in survivors of the acute respiratory distress syndrome. $N$ Engl J Med 2003; 348: 683-693.

26 Schelling G, Stoll C, Vogelmeier C, et al. Pulmonary function and health-related quality of life in a sample of long-term survivors of the acute respiratory distress syndrome. Intensive Care Med 2000; 26: $1304-1311$.
27 Ware LB, Koyama T, Billheimer DD, et al. Prognostic and pathogenetic value of combining clinical and biochemical indices in patients with acute lung injury. Chest 2010; 137: 288-296.

28 Calfee CS, Ware LB, Glidden DV, et al. Use of risk reclassification with multiple biomarkers improves mortality prediction in acute lung injury. Crit Care Med 2011; 39: 711-717.

29 Ware LB. Prognostic determinants of acute respiratory distress syndrome in adults: impact on clinical trial design. Crit Care Med 2005; 33: S217-S222.

30 Raymondos K, Martin MU, Schmudlach T, et al. Early alveolar and systemic mediator release in patients at different risks for ARDS after multiple trauma. Injury 2012; 43: 189-195.

31 Gharib SA, Liles WC, Klaff LS, et al. Noninjurious mechanical ventilation activates a proinflammatory transcriptional program in the lung. Physiol Genomics 2009; 37: 239-248.

32 Stern JB, Fierobe L, Paugam C, et al. Keratinocyte growth factor and hepatocyte growth factor in bronchoalveolar lavage fluid in acute respiratory distress syndrome patients. Crit Care Med 2000; 28: 2326-2333.

33 Miyazaki E, Nureki S, Ono E, et al. Circulating thymus- and activation-regulated chemokine/CCL17 is a useful biomarker for discriminating acute eosinophilic pneumonia from other causes of acute lung injury. Chest 2007; 131: 1726-1734.

34 Ranieri VM, Suter PM, Tortorella C, et al. Effect of mechanical ventilation on inflammatory mediators in patients with acute respiratory distress syndrome: a randomized controlled trial. JAMA 1999; 282: 54-61.

35 Checkley W, Brower RG, Munoz A. Inference for mutually exclusive competing events through a mixture of generalized gamma distributions. Epidemiology 2010; 21: 557-565. 